with respect to chronological age was observed in 10 cases. A very significant correlation was observed between BMI and vertebral BMD $(r=$ $0.75, p=0.000)$

Conclusion: The frequency and severity of the decline in BMD in children with $C D$ requires regular monitoring. It seems to be favored by a low BMI, which is common during $\mathrm{CD}$ in relation with the associated malabsorption.

Disclosure of Interests: None declared

DOI: 10.1136/annrheumdis-2019-eular.8108

\section{AB0827 IS DENOSUMAB SAFE IN HIV PATIENTS?}

Isabel de la Morena ${ }^{1,1}$, Sara Vela ${ }^{2}$, Juan Alberto Paz Solarte ${ }^{1}$, Diego Bedoya ${ }^{1}$, M Jose Galindo ${ }^{3} .{ }^{1}$ Hospital Clinico Universitario de Valencia, Rheumatology, Valencia, Spain; ${ }^{2}$ Hospital Clinico Universitario de Valencia, Internal Medicine, Valencia, Spain; ${ }^{3}$ Hospital Clinico Universitario de Valencia, Infectious Diseases, Valencia, Spain

Background: The survival of the infected patients by the human immunodeficiency virus (HIV) has increased in the last years, increasing the incidental comorbilities, being osteoporosis (OP) one of them, caused by traditional risk factors, by the ones associated to the infection itself and due to the specific treatments used. The denosumab is a human monoclonal antibody that acts against the link of the receptor that activates the nuclear kappa B (RANK-L), and among its secondary effects most commonly described, there are the infections, reason why its indication in HIV patients requires special caution.

Objectives: To analize efficacy, security and compliance to denosumab in HIV patients.

Methods: Retrospective longitudinal observational study. There were included all HIV patients with OP diagnosed by bone mineral density measured by dual-energy X-ray densitometry (DEXA) or fragility fractures treated with denosumab at Infectious Diseases Unit of our Hospital from August 2014 until December 2018. There were collected clinical data, immunological status, antiretroviral therapies, fragility fractures and duration, adverse events, tolerance and compliance to denosumab treatment.

Results: There were included 14 patients, 8 females and 6 males, with a mean age of 58.35 (50-73) years old, and a mean HIV evolution of 23,78 ( \pm 9.56) years, with a mean denosumab treatment duration of 27.07 (9-58) months. They presented a DEXA mean T-score values of -2.39 for femoral neck and -2.96 for spine. There were collected a patient with múltiple vertebral fractures, and an other patient with a single vertebral fractures before started denosumab treatment and 1 distal radius fracture 9 months on denosumab treatment. There were reported adverse events in 5 of the 14 patients: 2 mild infections of the upper respiratory track, 1 injection site rash that lead to the interruption of the treatment, and 2 exitus but none of them with a direct relationship with denosumab treatment: a patient with refractory small cell lung cancer history, deceased of pneumonia, and a 77 year old woman with Alzheimer disease deceased of respiratory infection. Compliance was not met in three patients, 1 because lost of follow-up, 1 caused by bad general compliance and another because indication of odontological surgery, these two last ones are waiting for re-introduction. There have not been collected jaw osteonecrosis neither atypical fractures cases. There have not been collected viral loads or CD4 changes during the follow-up period that involved a switch for the OP treatment. The antiretroviral treatment changes have been caused by intolerances to previous treatments and treatment simplifications in order to improve the compliance, but never because denosumab concomitant therapy.

Conclusion: Denosumab treatment in the HIV patients of our series is well tolerated, has adequate adherence and seems safe although perhaps it should be used with greater caution in patients with comorbidities.

Disclosure of Interests: Isabel de la Morena Speakers bureau: Abbvie, Celgene, Pfzier, UCB, Ghebro, Roche, Sanofi, Janssen., Sara Vela: None declared, Juan Alberto Paz Solarte Employee of: He is working at UCB since December 2018, when the patient recruitment ended., Speakers bureau: Abbvie, Roche, Pfzier, Novartis, Celgene, Amgen, MSD, Janssen, Diego Bedoya: None declared, M Jose Galindo: None declared

DOI: 10.1136/annrheumdis-2019-eular.7858

\section{AB0828 \\ RHEUMATIC PATHOLOGY AFTER SOLID ORGAN TRANSPLANTATION}

Madalina Duna ${ }^{1}$, Denisa Predeteanu ${ }^{2}$, Violeta Bojinca ${ }^{2}$, Ianos Pahomea ${ }^{3}$,

Nicolae Boleac ${ }^{3}$, Radu Poenaru ${ }^{3}$, Narcis Copca ${ }^{3} .{ }^{1}$ Romania, Internal Medicine and Rheumatology, Bucharest, Romania; " "Sfanta Maria" Hospital, Internal Medicine and Rheumatology, Bucharest, Romania; "Sfanta Maria" Hospital, Department of Surgery and Transplantation, Bucharest, Romania

Background: Organ transplantation has become as effective therapy for end-stage renal, hepatic, cardiac and pulmonary diseases within the past 2 decades. Osteoporosis, low level of vitamin $D$ and hyperuricemia have emerged as frequent and sometimes devastating complications of organ solid transplantation process.

Objectives: The objective of the study was to detect the prevalence of osteoporosis, low level of vitamin D and hyperuricemia in liver and lung transplant patients.

Methods: We evaluated a cohort of 48 patients who underwent liver and lung transplantation in a single Romanian center between July 2014 and January 2019. 44 pts undergone liver transplantation and 4 undergone lung transplantation. We have measured the level of the 25-hydroxyvita$\min \mathrm{D}$ and serum urate before and after the transplantation in all patients.

Results: The average age of the patients was $53.7 \pm 10$ years (range $32-69)$ with a sex ratio $1: 1.32(66 \%)$ of patients came from urban area. $8(16.6 \%)$ patients were excluded from this study due to recurrent HCV infection with hepatic failure in the first few months after transplantation. The final group consisted of 40 patients.

The indications of the orthotopic liver transplantation in these patients were: cirrhosis secondary chronic hepatitis B, C, D virus infection in 24 $(60 \%)$ cases, autoimmune hepatitis in $7(17.5 \%)$ patients, alcoholic liver disease in $2(5 \%)$ patients, hepatocellular carcinoma in $1(2.5 \%)$ patient, polycystic liver disease in $1(2.5 \%)$ patient and nonalcoholic fatty liver disease in 1 (2.5\%) patient.

In all 4 cases with lung transplantation, the reason for of the lung transplantation was chronic obstructive pulmonary disease (COPD).

$36(91 \%)$ patients were receiving combination immunosuppressant therapy (tacrolimus and) and only $4(9 \%)$ patients were receiving cyclosporine; in this situation, the association between tacrolimus and mycophenolate mofetil was preferred versus cyclosporine because of the negative effect of cyclosporine on the bone.

Post-transplant, hyperurcemia (according to local laboratory reference values $>6.5 \mathrm{mg} / \mathrm{dL}$ ) was presented in $28(70 \%)$ transplant recipients but none of the hyperuricemic patients developed gout. Despite this fact, all of them were treated with allopurinol.

Vitamin D deficiency was reported in $22(55 \%)$ transplant recipients. Also, a DXA scan of the lumbar spine and femoral neck measuring T-score and bone mineral density (BMD) was routinely performed after a mean interval of 4-6 months post-transplant. From this group of patients, 10 (25\%) patients had decreased bone mineral density ( $<2.5$ SD) and 8 $(20 \%)$ of them who complained of back pain in the early posttransplantation period, presented on $\mathrm{x}$-rays vertebral fragility fractures at different sites (most frequently in the thoracic spine). The mean T-score was -2.7 and the mean BMD was $27.4 \mathrm{~kg} / \mathrm{m}^{2}$.

Conclusion: The standard care of liver and lung transplant recipients must includes, besides other lab tests, a measurement of 25-hydroxyvita$\min D$ and serum urate. For patients with low levels of 25-hydroxyvitamin D, evaluation for osteoporosis (risk factors, x-ray, DXA scan) should be mandatory.

\section{REFERENCES}

[1] P. R. Ebeling, "Approach to the patient with transplantation-related bone loss," The Journal of Clinical Endocrinology \& Metabolism, vol. 94, no. 5, pp. 1483-1490, 2009.

[2] Sullivan PM, William A, tichy EM, "Hyperuricemia and gout in solid-organ transplant: update in pharmacological management," Prog Transplant. 2015 Sep;25(3):263-70. doi: 10.7182/pit2015322.

[3] Mazzali M, "Uric acid and transplantation", Semin Nephrol. 2005 Jan;25 (1):50-5.

Disclosure of Interests: Madalina Duna: None declared, Denisa Predeteanu: None declared, Violeta Bojinca Speakers bureau: multiple, lanos Pahomea: None declared, Nicolae Boleac: None declared, Radu Poenaru: None declared, Narcis Copca: None declared DOI: 10.1136/annrheumdis-2019-eular.6087 